Learning Outcomes Our program highlights the feasibility and acceptability of an intervention for the secondary prevention of partner violence. A wider roll out of this program would directly contribute to the achievement of 6 out of the 17 Sustainable Development Goals.

\section{E.002 SEX-SPECIFIC AND AGE-SPECIFIC SUICIDE MORTALITY BY METHOD IN 58 COUNTRIES, 2000-2015}

${ }^{1}$ Yue Wu*, ${ }^{2}$ David Schwebel, ${ }^{1}$ Yun huang, ${ }^{3}$ Peishan Ning, ${ }^{3}$ Peixia Cheng, ${ }^{3}$ Guoging Hu. ${ }^{1}$ Department of Occupational and Environmental Health, Xiangya School of Public Health, Central South University, Changsha, China; ${ }^{2}$ Department of Psychology, University of Alabama at Birmingham, Birmingham, USA; ${ }^{3}$ Department of Epidemiology and Health Statistics, Xiangya School of Public Health, Central South University, Changsha, China

10.1136/injuryprev-2021-safety. 179

Background Suicide is a significant public health problem internationally. Recent suicide mortality by method is unexamined.

Methods Using mortality data from the WHO mortality database, we compared sex-, age-, and country-specific suicide mortality by method for 58 countries worldwide between 2000 and 2015. Changes in suicide mortality were quantified using negative binomial models among three age groups for males and females separately.

Results Suicide mortality declined substantially for both sexes and all three age groups studied in 37 of the 58 included countries between 2000 and 2015. Males consistently had much higher suicide mortality rates than females in all 58 countries. Hanging was the most common suicide method in the majority of countries. Sex-specific suicide mortality varied across the countries significantly for all three age groups. The spectrum of suicide method generally remained stable for 28 of the 58 included countries; notable changes occurred in the other 30 countries, including especially Colombia, Finland and Trinidad and Tobago.

Conclusion Likely as a result of prevention efforts as well as sociodemographic changes, suicide mortality decreased substantially in 37 of the included 58 countries between 2000 and 2015. Further action is recommended to explore specific drivers of recent changes (particularly for increasing suicide rates in eight countries), to understand substantial disparities in suicide rates across countries, and to develop interventions to reduce suicide rates globally.

Learning Outcomes Between 2000 and 2015, suicide mortality decreased in 37 countries but increased in 8 . Suicide spectrum by method experienced substantial changes over time in several countries.

\section{E.003 HEALTH CARE PROVIDERS' PERCEPTIONS OF THE BARRIERS TO SUICIDE PREVENTION IN MEXICO}

${ }^{1}$ Lourdes Gómez-García*, ${ }^{2}$ Marcela Agudelo-Botero, ${ }^{3}$ Maria de la Luz Arenas-Monreal, ${ }^{2}$ Mario Rojas-Russell, ${ }^{3}$ Rosario Valdez-Santiago. 'Programa de Maestría y Doctorado en Ciencias Médicas, Odontológicas y de la Salud, Facultad de Medicina, Universidad Nacional Autónoma de México, Mexico; ${ }^{2}$ Centro de Investigación en Políticas, Población y Salud, Facultad de Medicina, Universidad Nacional Autónoma de México, Mexico; ${ }^{3}$ Centro de Investigación en Sistemas de Salud, Instituto Nacional de Salud Pública, Cuernavaca, Mexico

10.1136/injuryprev-2021-safety. 180

Background Suicide is a growing public health issue around the world. Despite its importance, few countries have established national programs to address suicide prevention. In middle-income countries, few studies focus on the factors that deter mental health care provision on suicide prevention. This qualitative study aimed to explore mental health care providers' perceptions of barriers to suicide prevention in Mexico.

Methods Semi-structured interviews were conducted with 25 mental health care providers who worked in outpatient mental health care units in Mexico City. Data were analyzed using framework analysis.

Results Three broad categories were identified: structural issues in service provision, health care services issues and social issues of the patient. Participants tough that the lack of a suicide program, mental health system fragmentation and the poor attention of the authorities to mental health hinders patient's access to appropriate health care. Insufficient resources, service's saturation and poor capacitation difficult opportune detection and treatment. Social problems as violence, drug use, and family disintegration are leading the patients to suicide behavior.

Conclusions Mental health issues are not a priority in the national policy agenda. The absence of a national policy to suicide prevention leads to poor resource allocation and ineffective actions to prevent suicide. Our findings suggest that suicide prevention efforts may promote intersectoral collaboration, more integrated health services, and considering social issues to achieve a comprehensive approach.

\section{E.004 VIOLENCE REDUCTION UNITS: PUBLIC HEALTH APPROACH TO PREVENTING VIOLENCE (ENGLAND AND WALES)}

Zara Quigg* , Hannah Timpson, Nadia Butler, Matthew Millings, Rebecca Harrison. Liverpool John Moores University, LIVERPOOL, UK

\subsection{6/injuryprev-2021-safety. 181}

Background In 2018, UK Government published its Serious Violence Strategy in response to increases in knife and gun crime. The strategy declared a call to action for partners across different sectors to collaborate and adopt a whole system public health approach to preventing serious violence. In June 2019, 18 areas were provided with funding to establish violence reduction units (VRUs), bringing together police, local government, health, community leaders and other key partners with a specific intent to tackle violence and its underlying causes. This study aims to identify the development and implementation of VRUs during 2019/20, including facilitating and moderating factors, and outcomes and impacts.

Methods The study focuses on three VRU areas across England and Wales (population $\sim 5$ million). Methods include interviews $(n \sim 40)$ and engagement (e.g. via workshops; $n \sim 200)$ with a range of stakeholders (e.g. VRU team members/advisors; intervention programme commissioners/delivers), analyses of programme documentation, and observation of programme delivery.

Results Initial findings illustrate that the three VRUs are implementing a place-based approach to violence prevention, have developed a problem profile and strategy for future prevention activity (in the short and long-term), and implemented various interventions to prevent and respond to violence. Interventions target system change, and also consider a life course public health perspective. 
Conclusion/Learning Outcomes Key learning from the initial development and implementation of the VRUs will be presented, considering the principles of the public health approach and UK government guiding principles for VRUs: collaboration; co-production; cooperative in data and intelligence sharing; counter-narrative development; and community consensus.

\section{E.005 FINDING SUBSTANCE USE DISORDER TREATMENT FACILITY OPENINGS: NATIONAL EXPANSION OF KENTUCKY'S LOCATOR}

Terry Bunn*, Tyler Jennings, Catherine Hines, Danita Coulter, Jodie Weber, Amber Kizewski. University of Kentucky, Lexington, USA

\subsection{6/injuryprev-2021-safety. 182}

Context FindHelpNowKY.org (FHNKY) is a drug overdose prevention website intervention developed and implemented in 2018 to timely link individuals to substance use disorder (SUD) treatment facilities with available openings in the Commonwealth of Kentucky. FHNKY is a near real time treatment locator where facilities update their openings on a regular basis. In February 2020, there were 607 facilities who participated on FHNKY and have updated their availability over 11,000 times; over 142,000 searches were performed. The website is used by health professionals, safety professionals, friends and family, as well as individuals themselves in search of SUD treatment.

Process The FHNKY website was rebranded as a national FindHelpNow (FHN) platform that includes standardized national search criteria, and specifically branded marketing materials.

Analysis A FHN peer-to-peer learning network was established for other states to utilize the national FHN platform and/or FHNKY strategies and approaches.

Outcomes Fourteen interested states attended an in-person meeting with multiple presentations on website development, and functionality in November 2019. In February 2020, five states and jurisdictions adopted the FHN platform and recruitment strategies, and memorandums of understanding have been established.

Learning Outcomes This initial development and implementation phase of a national standardized FHN platform will enhance the ability of any individual anywhere in the U.S., who seeks SUD treatment at their critical stage of readiness, to find a SUD treatment facility that accepts their form of payment (or fee-free), and provides necessary services such as the provision of medication for opioid use disorders and concurrent mental health treatment.

\section{A - Road - Programs, March 25, 2021}

\section{A.001 ONE-SIDEDNESS OF MOTORCYCLE HELMET USE: A CHALLENGE TO MOTORCYCLE SAFETY IN NEPAL}

${ }^{1}$ Puspa Raj Pant*, ${ }^{2}$ Lennart Hellmann, ${ }^{3}$ Hanhe Lin, ${ }^{2}$ Rüdiger Trimpop, ${ }^{4}$ Felix Siebert. ${ }^{1}$ University of the West of England Bristol, Bristol, UK; ${ }^{2}$ University of Jena, Jena, Germany; ${ }^{3}$ University of Konstanz, Konstanz, Germany; ${ }^{4}$ Technische Universität Berlin, Berlin, Germany

10.1136/injuryprev-2021-safety. 183
Background Mandatory motorcycle helmet use regulation is essential, but its enforcement is even more important for head injury prevention, especially in a country like Nepal with a high share of motorcycle traffic. We assessed the impact of one-sided motorcycle helmet use regulation in Nepal, where helmet use is mandatory, but only drivers are fined for not using a helmet, while passengers do not get punished.

Methods In a combined observational and questionnaire survey, knowledge about the helmet use regulation was assessed and adherence to the law evaluated. Data was collected at seven sites throughout the country, including rural and urban regions.

Results The observation of 2548 motorcycle riders reveals a dichotomy of motorcycle helmet use in Nepal. While $98 \%$ of drivers used a helmet, only less than $1 \%$ of observed passengers used a helmet. The questionnaire survey of 220 riders reveals a lack of knowledge about the existing mandatory helmet use for passengers, most probably due to inadequate enforcement of the helmet rule for them. The survey further reveals that helmets would be readily available for passengers, as over $50 \%$ have access to a helmet.

Conclusion In Nepal, the helmet use of motorcycle passengers is critically low. The sole existence of rules for mandatory helmet use is not enough, regulatory changes in the traffic-fine structure are necessary to increase helmet use and prevention of consequences of head injuries.

Learning Outcomes Educating motorcycle passengers about existing rules and benefits of using a standard helmet may support motorcycle safety in Nepal.

\section{A.002 COST OF MOTORCYCLE CRASH VICTIMS AT A PUBLIC TERTIARY HEALTHCARE FACILITY IN KARACHI, PAKISTAN}

${ }^{1}$ Asrar Ali* ${ }^{2}$ Adnan Hyder, ${ }^{1}$ Uzma Rahim Khan, ${ }^{3}$ Muhammad Ashar Malik. ${ }^{1}$ Aga Khan University Hospital, Emergency Medicine, Karachi, Pakistan; ${ }^{2}$ George Washington University Milken Institute School of Public Health, USA; ${ }^{3}$ Aga Khan University, Medical College, Community Health Sciences, Karachi, Pakistan

\subsection{6/injuryprev-2021-safety. 184}

Background Road traffic injuries account for the major proportion of injury and economic burden globally. However, motorcyclists are the most vulnerable group to sustain fatal and non-fatal injuries. The use of helmet is one of most acknowledged protective measure. However cost-saving ability of helmet use is inconsistent in the literature.

Aim To estimate the difference in the healthcare cost of motorcycle crash in those who were wearing helmet and those who were not wearing helmet in Karachi, Pakistan.

Methodology We conducted a cross-sectional study by using micro-costing method and out-of-pocket expenditure data collection for healthcare cost estimation in a public tertiary hospital of Karachi, Pakistan. We included 18 year and above victims of motorcycle crash both riders and pillions through consecutive sampling from the emergency department and grouped them in to helmeted and non-helmeted. Cost differences were compared using t-test.

Result We recruited 323 participants, 127 (39\%) were wearing helmet, while, 93 (60\%) were not wearing helmet at the time of crash. The helmeted group had $18 \%$ head injuries as compared to $46 \%$ in the non-helmeted. Helmeted group incurred a total healthcare cost of PKR.15855, whereas, nonhelmeted victims had a median healthcare cost of 